\title{
NUEVOS REGISTROS DE QUIRÓPTEROS PARA LA RESERVA NACIONAL DE PARACAS, PERÚ
}

\section{NEW RECORDS OF BATS FOR PARACAS NATIONAL RESERVE, PERU}

\author{
Francesca G. Montero Commisso ${ }^{1}$, Carla Gazzolo Navarro ${ }^{2}$, Gabriella Gonzalez Blacker ${ }^{1}$
}

\section{Resumen}

Se registró la presencia de Glossophaga soricina (Pallas 1766) (Phyllostomidae) y Myotis atacamensis (Lataste 1957) (Vespertilionidae) dentro de la Reserva Nacional de Paracas. Dichas especies no están actualmente incluidas en la lista de mamíferos del Plan Maestro 2003-2007 de dicha Reserva. Ambas especies han sido reportadas para el departamento de Ica. Se debe resaltar que Myotis atacamensis (Lataste, 1957) se encuentra como Especie Vulnerable según la UICN.

Palabras clave: Glossophaga soricina, Myotis atacamensis, Reserva Nacional de Paracas, registros nuevos

\begin{abstract}
The presence of Glossophaga soricina (Pallas 1766) (Phyllostomidae) and Myotis atacamensis (Lataste 1957) (Vespertilionidae) within the Paracas National Reserve was registered. Currently, these species are not included in the mammals list of the Reserve Master Plan 2003-2007. Both species have been reported for the Department of Ica. In addition, it must be highlighted that Myotis atacamensis (Lataste, 1957) is categorized as Vulnerable according the IUCN.
\end{abstract}

Key words: Glossophaga soricina, Myotis atacamensis, Reserva Nacional de Paracas, new records

\section{Introducción}

La lista de mamíferos de la Reserva Nacional de Paracas, detallada en el Plan Maestro 2003-2007 (INRENA, 2002), incluye 5 especies de mamíferos terrestres. Dos de éstas corresponden a quirópteros Desmodus rotundus (E. Geoffroy Sain-Hilaire 1810) y Platalina genovensium (Thomas 1928).

En el mes de Febrero 2006, como parte del Estudio de Línea Base para la Ampliación de la Planta de Fraccionamiento de Gas, se realizó una evaluación rápida de quirópteros. Se capturaron dos especies de murciélagos Glossophaga soricina (Pallas 1766) y Myotis atacamensis (Lataste 1957) dentro de la Reserva Nacional de Paracas.

\section{Materiales y Métodos}

Las capturas se produjeron en el área denominada Oasis de Paracas. Este hábitat está ubicado en las coordenadas $13^{\circ} 50^{\prime} 24^{\prime \prime} \mathrm{S}$ y $76^{\circ} 12^{\prime} \mathrm{W}$ al norte de la Reserva Nacional de Paracas (Figura 1).

Esta área posee una extensión aproximada de 6 ha y presenta 3 cuerpos de agua, que sustentan dicha formación. El oasis limita con los sectores de recuperación de Santa Cruz y con la zona silvestre del desierto (INRENA, 2002).

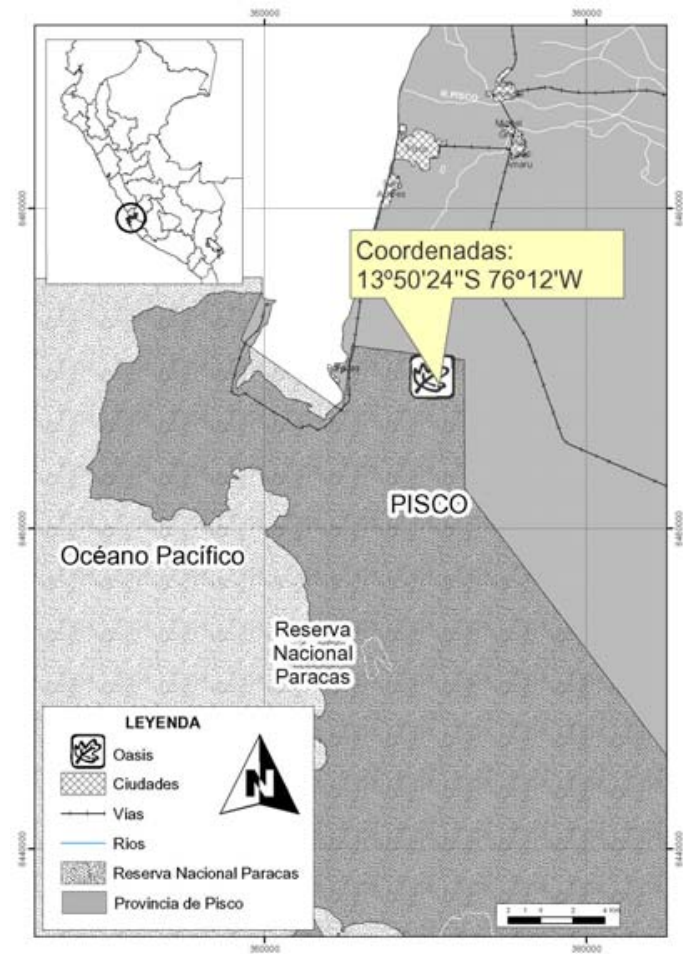

Figura 1. Mapa de ubicación de la zona de evaluación

Esta formación vegetal es única y peculiar en el área del semidesierto. Se presenta como una 
comunidad arbórea, cuya especie dominante es Geoffroea decorticans (Gillies ex Hook. \& Arn. Burkart 1833) “sofaique” o “chañar”. En general, la vegetación es de hábito herbáceo. Considerando el porcentaje de cobertura predominan las especies Sporobolus virginicus y Spilanthes leiocarpa (Gonzalez, en prep.).

Para la captura de murciélagos se colocaron redes de neblina. El esfuerzo total de muestreo fue de 243 $\mathrm{m}^{2}$ de red a lo largo de 33 horas distribuidas en tres noches. Se marcó a los individuos capturados para considerar la eventual recaptura, asimismo se liberó a los individuos cuya especie se pudo determinar in situ. La especie no definida se colectó para su posterior identificación. Dicho proceso se llevó a cabo en el laboratorio de Fisiología Animal y Biorremediación "Luis Basto Acosta” del Departamento Académico de Biología de la Universidad Nacional Agraria La Molina.

\section{Resultados y Discusión}

Se capturaron un total de 4 individuos, los cuales corresponden a dos especies. Tres especimenes resultaron ser Glossophaga soricina (Pallas 1766) (Phyllostomidae) y uno Myotis atacamensis (Lataste 1957) (Vespertilionidae). No se produjeron recapturas (Figuras 2 y 3).

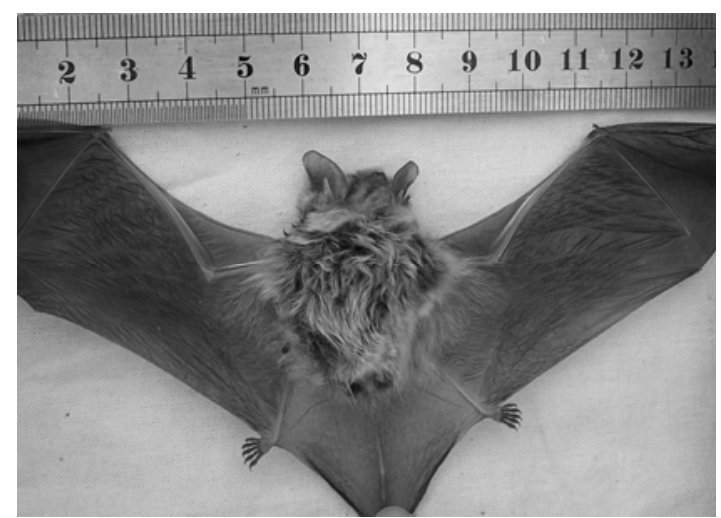

Figura 2. Myotis atacamensis (Lataste 1957)

Ambas especies han sido mencionadas como presentes en el Departamento de Ica. La especie $G$. soricina fue registrada en construcciones urbanas, huertos de árboles frutales y en la vegetación del monte ribereño. Mientras que $M$. atacamensis fue encontrada en la vegetación de huertos, chacras y entre el follaje de árboles y arbustos (Hernandez-Ríos \& Velásquez, 1998).

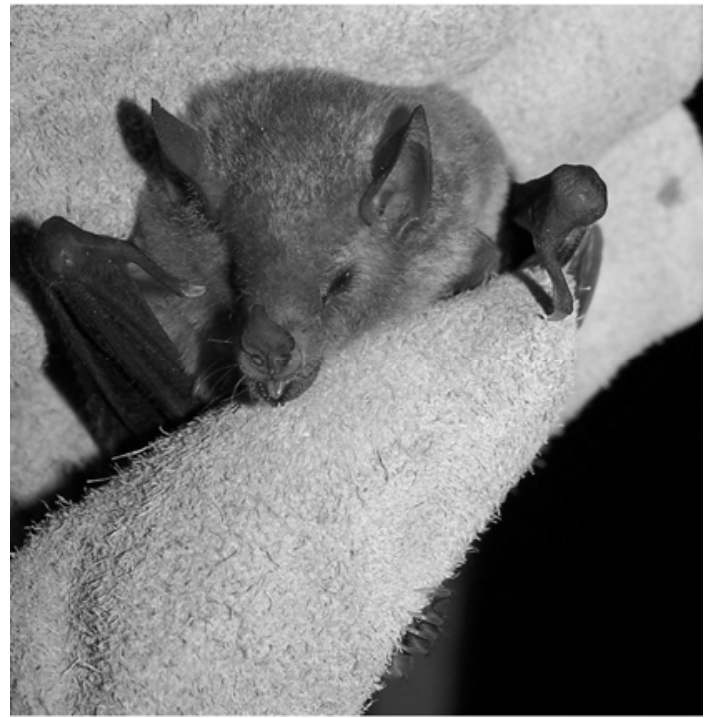

Figura 3. Glossophaga soricina (Pallas 1766)

La vegetación del Oasis y de los campos de cultivo aledaños constituiría una importante fuente de alimento para los murciélagos frugívoros e insectívoros. Se presentan palmeras datileras (Phoenix dactylifera) y algarrobos (Prosopis pallida). Es probable que estos murciélagos sean importantes polinizadores y dispersores de semillas de estos árboles.

Los hábitos alimentarios de $G$. soricina incluyen fruta, néctar y polen, provenientes de gran variedad de plantas (Alvarez \& Sanchez-Casas, 1999), y partes de flores (Mena \& Williams, 2002). Adicionalmente, se han identificado insectos en su dieta (Rojas et al., 2005; Gazzolo \& Montero, en prep.), entre ellos algunas especies del Orden Orthoptera y/o Coleoptera (Gazzolo \& Montero, en prep.). La dieta de $M$. atacamensis se indica como únicamente insectívora (Hernández-Ríos \& Velásquez, 1998), usual para todas las especies de la familia Vespertilionidae (Nowak, 1994).

Se sabe además que las familias Phyllostomidae y Vespertilionidae son más abundantes en hábitats no disturbados (Solari et al., 2002). Por lo tanto, la presencia de $G$. soricina y $M$. atacamensis podría considerarse como indicadora de disturbios en un hábitat como el Oasis de Paracas. Adicionalmente, el Orden Chiroptera ha sido propuesto como indicador de niveles de perturbación de hábitat (Hernandez-Ríos \& Velásquez, 1998). La importancia del registro de estas dos especies radica en el hecho de no estar incluidas en la lista de esta Área Natural Protegida (ANP). Además, se debe tener presente que $M$. atacamensis se encuentran bajo la categoría Vulnerable, según la UICN (2007). 


\section{Conclusiones}

Se añaden dos nuevos registros de murciélagos dentro de la Reserva Nacional de Paracas, G. soricina y $M$. atacamensis. Estas especies deberían incluirse en la lista de mamíferos del Plan Maestro de esta ANP. El haber encontrado dos especies de murciélagos en esta evaluación rápida indica que pueden estar presentes otras especies aún no incluidas en dicha lista. El Oasis podría resultar un buen lugar de evaluación por su condición de refugio en el desierto.

\section{Agradecimientos}

Agradecemos a Environmental Resources Management Perú (ERM), en especial a Francisco Pinilla, Julio Arenas y Daniel Takahashi por el apoyo brindado para la realización del presente estudio. Al Laboratorio de Fisiología Animal y Biorremedicación "Luis Basto Acosta” del Departamento Académico de Biología de la UNALM por la ayuda con la identificación de los especimenes. Al INRENA y a la Reserva Nacional de Paracas por el permiso otorgado y el apoyo brindado a las autoras. A Marta Williams de Castro, revisora del manuscrito.
Alvarez T \& N. Sanchez-Casas. 1999. Diferenciación alimentaria entre los sexos de Glossophaga soricina (Chiroptera:Phyllostomidae) en México.

Gazzolo C. y Montero F.G. (En prep.). Artrópodos en la dieta de Glossophaga soricina.

Gonzalez G. (En prep.) Cap. Vegetación en: Libro de Biodiversidad de Pisco.

Hernandez-Ríos M \& V. Velásquez. 1998. Estudio de los Murciélagos del Valle de Ica. Boletín de Lima. 111:5462.

Instituto Nacional de Recursos Naturales (INRENA). 2002. Reserva Nacional de Paracas - Plan Maestro 2003 2007.

Mena J. \& M. Williams de Castro. 2002. Diversidad y patrones reproductivos de quirópteros en un área urbana de Lima. Perú. Ecología Aplicada 1(1): 1-8.

Nowak R. 1994. Walker's Bats of the World. The Johns Hopkins University Press. Baltimore, Maryland, y London, U.K.

Solari S., J.J. Rodríguez, E. Vivar \& P.M. Velazco. 2002. A framework for assessment and monitoring of small mammals in a lowland tropical forest. Environmental Monitoring and Assesment 76: 89-104.

UICN 2007. The 2007 IUCN Red List of Threatened Species-www.iucnredlist.org/

\section{Literatura citada}

${ }^{1}$ Environmental Resources Management Perú S.A. (ERM). Felipe Pardo y Aliaga Nº40 Of. 1120 San Isidro. galia_mc@yahoo.es, gabriella.gonzalez@erm.com

${ }^{2}$ Laboratorio de Fisiología Animal, Universidad Nacional Agraria La Molina. Av. La Molina s/n, La Molina. carlagazzolo@yahoo.es 